\title{
The Dark Purple Side of Ceftriaxone: A Case Report on Leucocytoclastic Vasculitis
}

\author{
Elia Rigamonti ${ }^{1}$, Francesca Bedussi ${ }^{2}$, Jerome Blanc ${ }^{1}$, Pietro Gianella ${ }^{1}$, Gianluca Vanini ${ }^{1}$ \\ ${ }^{1}$ Service of Internal Medicine, Ospedale Regionale di Lugano (Italiano), Ente Ospedaliero Cantonale, Lugano, Switzerland \\ ${ }^{2}$ Division of Clinical Pharmacology and Toxicology, Institute of Pharmacological Sciences of Southern Switzerland, Ente Ospedaliero Cantonale, Lugano,
} Switzerland

Received: 03/01/2020

Accepted: $16 / 01 / 2020$

Published: 05/03/2020

How to cite this article: Rigamonti E, Bedussi F, Blanc J, Gianella P, Vanini G. The dark side of ceftriaxone: a case report on leucocytoclastic vasculitis. EJCRIM 2020;7: doi:10.12890/2020_001464.

Conflicts of Interests: The Authors declare that there are no competing interests.

This article is licensed under a Commons Attribution Non-Commercial 4.0 License

\section{ABSTRACT}

We present a case of an 85-year-old woman diagnosed with uncomplicated pyelonephritis, who was treated with intravenous ceftriaxone. Her chronic medications were phenprocoumon, diltiazem and bisoprolol. During the infectious phase, the patient presented tachycardia - despite high-dose beta-blocker treatment - and developed left acute heart failure, with acute renal failure (pre-renal origin). After introduction of furosemide diuretic therapy, clinical conditions improved and better control of the volemic status and heart rate was achieved. Several days after ceftriaxone and digoxin therapy initiation, worsening multiple non-blanching palpable purpuric lesions with bullae and papules, limited to the lower extremities, were noted. Skin biopsy was performed and a diagnosis of leucocytoclastic vasculitis, with associated panniculitis, was made. Ceftriaxone was discontinued and systemic corticosteroids were introduced, with a clear improvement in the cutaneous condition.

\section{LEARNING POINTS}

- Leucocytoclastic vasculitis is a rare but significant side effect related to the administration of ceftriaxone.

- The importance of skin biopsy in the differential diagnosis of skin eruptions.

\section{KEYWORDS}

Vasculitis, ceftriaxone, dermatology

\section{CASE DESCRIPTION}

An 85-year-old woman, with known permanent atrial fibrillation, moderate mitral insufficiency and chronic renal failure, presented to the Emergency Room after an accidental fall. Her chronic medications were phenprocoumon (for at least 7 years), diltiazem and bisoprolol. The patient reported dysuria. Haematological examination showed leucocytosis and increased inflammatory markers. The urinary sediment was pathologic (leucocyturia, bacteriuria). The urinary culture was positive for multi-sensitive Escherichia coli. Abdominal CT showed dilatation in the right pyelocalyceal cavities. Uncomplicated pyelonephritis was diagnosed, and intravenous antibiotic therapy with ceftriaxone was started.

During the infectious phase, the patient presented tachycardia (up to $160 \mathrm{bpm}$ ), despite high-dose treatment with beta blockers. She developed left acute heart failure, with acute on chronic renal failure (pre-renal origin). After introduction of diuretic therapy with furosemide, clinical conditions improved and better control of the volemic status and heart rate was achieved. 
Four and 5 days, respectively, following ceftriaxone and digoxin therapy initiation, worsening multiple non-blanching palpable purpuric lesions with bullae and papules, limited to the lower extremities, were noted (Fig. 1). Skin biopsy was performed and a diagnosis of leucocytoclastic vasculitis, with associated panniculitis, was made (Fig. 2).

Ceftriaxone was discontinued (after 7 days of therapy) and systemic corticosteroids were introduced, with a clear improvement in the cutaneous condition.

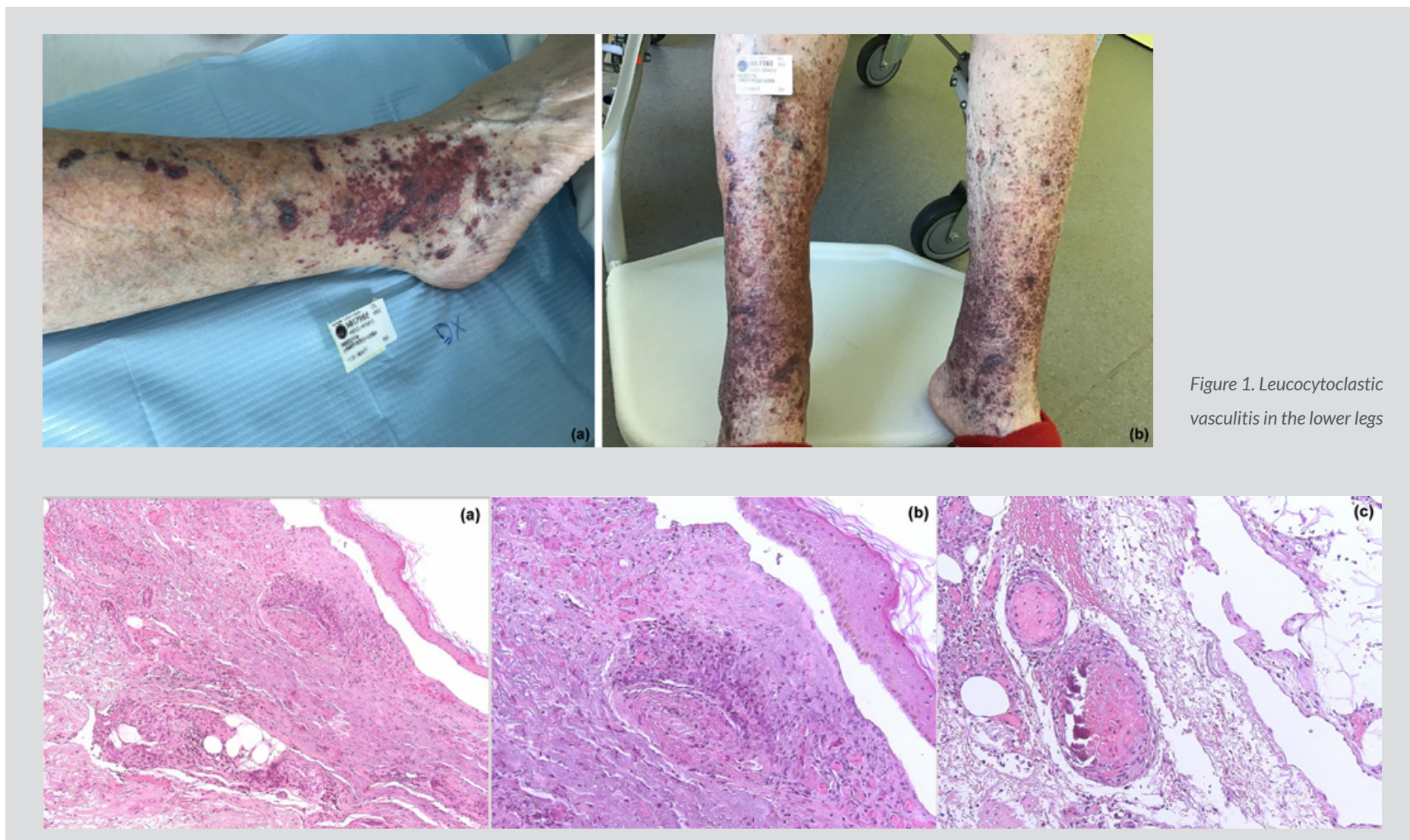

Figure 2. Skin biopsy. A: Panoramic view with severe vasculitis, B: detailed vascular structure and C: deep dermis and hypodermis tissue, with another vessel affected by vasculitis

\section{DISCUSSION}

In our case, the most interesting aspect was the diagnostic challenge before the skin biopsy result. A systemic vasculitis was excluded; immunological blood tests did not yield any significant results.

We considered a drug reaction: digoxin and ceftriaxone were started after hospital admission concomitant with skin lesion appearance, but the clinical features were more suggestive of phenprocoumon involvement.

The first clinical suspicion was "late-onset warfarin-induced skin necrosis", a rare complication of therapy with warfarin and coumarin derivatives. It usually appears 3 to 6 days after initiation of therapy, but cases of late onset have been reported. A drug-associated recurrent skin necrosis in a 57-year-old patient was described with phenprocoumon ${ }^{[1]}$. The time of late onset, in case series, is from 15 days to 4 years ${ }^{[2]}$. This hypothesis did not appear to completely align with our patient's clinical features, as, independently of the time of onset, typical warfarin-induced skin necrosis manifestations are purpuric areas that have evolved into large, tender haemoserous bullae, which break down to form ulcers.

The second clinical suspicion was that ceftriaxone may have acted to enhance the anticoagulant effects of the vitamin $\mathrm{K}$ antagonist by cephalosporin-associated platelet inhibition and by decreasing the gastrointestinal tract flora. Our patient, moreover, had an inadequate diet, without the required vitamin $\mathrm{K}$ intake. The INR increased to 6.1 concomitantly with skin lesion appearance.

We finally considered the hypothesis of a reaction due to recently introduced drugs: digoxin has not been shown to produce similar reactions ${ }^{[3]}$, whereas ceftriaxone-induced leucocytoclastic vasculitis has been described ${ }^{[3]}$. 


\section{REFERENCES}

1. Xin C, Hu D, Li M. Late onset warfarin-induced skin necrosis. G Ital Dermatol Venereol 2019;154(2):205-208.

2. Essex DW, Wynn SS, Jin DK. Late-onset warfarin-induced skin necrosis: case report and review of the literature. Am J Hematol 1998;57(3):233-237.

3. Haehn DA, Patel A, Youngberg G, Gonzalez-Estrada A. Ceftriaxone-induced leucocytoclastic vasculitis. BMJ Case Rep 2019;12(4):e229411. 